\title{
Comparison of peak oxygen consumption during cycle and treadmill exercise in severe chronic obstructive pulmonary disease
}

\author{
R S Mathur, S M Revill, D D Vara, R Walton, M D L Morgan
}

\begin{abstract}
Background-In normal subjects treadmill exercise usually produces the greatest maximal oxygen consumption $\left(\mathrm{VO}_{2} \max \right)$. This may not be true for patients with severe chronic obstructive pulmonary disease (COPD) in whom bicycle exercise, which offers support for the shoulder girdle, may produce a higher oxygen consumption than treadmill exercise. The aim of this study was to determine which mode of exercise produced the greatest oxygen consumption in patients with severe COPD.

Methods - Eight patients with severe COPD (forced expiratory volume in one second $\left(F E V_{1}\right)$ more than three standardised residuals below predicted) exercised to a symptom limited maximum on a bicycle and on a treadmill on separate days. The workload on the bicycle was increased by 10 watts each minute, and the treadmill gradient was increased by $2 \cdot 5 \%$ alternate minutes whilst the speed remained constant. Measurements of oxygen consumption $\left(\dot{V}_{\mathrm{O}_{2}}\right)$, ventilation ( $\left.\dot{\mathrm{V}} \mathrm{E}\right)$, heart rate, and oxygen saturation were made, and capillary blood gases were measured before and immediately after exercise. Lactate concentration was measured before and four minutes after exercise.

Results - There were no differences at peak exercise between the two forms of exercise for $\dot{V}_{2}$ (median 11.7 and $12.2 \mathrm{ml} / \mathrm{min} / \mathrm{kg}$ for bicycle and treadmill, respectively), for VE (median 26.6 and $25 \cdot 0 \mathrm{l} / \mathrm{min}$, respectively), and for heart rate (median 119 and 115 beats $/ \mathrm{min}$, respectively). The median lactate levels after bicycle exercise were higher than those after the treadmill (2.42 $v 0.94 \mathrm{mmol} / \mathrm{l})$.
\end{abstract}

Conclusions - Although only a small number of patients was studied and individual variability was large, there was no clear difference between the two forms of exercise. Regular bicycle exercise was unfamiliar to this group of patients and generated the greatest lactate response. The results do not support the hypothesis that bicycle exercise will produce a better performance in patients with severe COPD, but the two modes of exercise cannot be used interchangeably.

(Thorax 1995;50:829-833)

Keywords: bicycle ergometer, treadmill, chronic obstructive pulmonary disease, exercise.
The assessment of disability in patients with chronic obstructive pulmonary disease (COPD) usually requires some estimate of exercise capacity. Since measures of impairment such as forced expiratory volume in one second $\left(\mathrm{FEV}_{1}\right)$ cannot predict exercise performance with any accuracy, it should be measured directly. ${ }^{12}$ Methods of assessing whole body exercise performance include tests of maximal capacity and endurance capacity. In health the maximal capacity for oxygen uptake during incremental exercise $\left(\mathrm{Vo}_{2} \mathrm{max}\right)$ is determined by the upper limit of oxygen delivery to respiring muscle. In severe lung disease a true $\dot{\mathrm{Vo}}_{2} \mathrm{max}$ is seldom achieved and peak performance $\left(\mathrm{V}_{2}\right.$ peak) can be limited by pulmonary ventilation. ${ }^{3}$

Maximal incremental exercise tests are usually performed on a cycle ergometer or a motorised treadmill. The advantages of the cycle ergometer include the ability to measure workload and the provision of a more stable platform for metabolic measurement. The treadmill, however, has the advantage of being a familiar form of exercise which can generate a $\dot{\mathrm{Vo}}_{2} \mathrm{max}$ up to $7 \%$ greater than the cycle ergometer. ${ }^{4}$ Evidence from patients with lung disease has suggested that the two forms of exercise are broadly equivalent in patients with mild or moderate impairment of $\mathrm{FEV}_{1}{ }^{5}$ However, few data are available of the comparative measurements in patients with severe COPD.

It is possible that patients with severe COPD may find, conversely, that bicycle exercise is easier to achieve than unsupported treadmill exercise. On the cycle ergometer the upper limb muscles are supported and may prevent destabilisation of the respiratory muscles which may contribute to a poor performance. ${ }^{6}$ The physical support of the upper limbs may actually improve overall performance - for example, higher work rates, greater oxygen consumption, lower Borg scores. Determination of the greatest oxygen consumption is important for repeat measurements following pharmacological intervention or following a period of exercise training and, particularly for this category of patient, if it is used to assess suitability for pulmonary rehabilitation. We have tested the hypothesis that patients with severe COPD could generate a higher oxygen consumption on the cycle ergometer than by unsupported treadmill exercise. 
Table 1 Anthropometric and resting lung function data with standardised residual values in the eight subjects

\begin{tabular}{|c|c|c|c|c|c|c|c|c|}
\hline $\begin{array}{l}\text { Subject no. } \\
(M / F)\end{array}$ & $\begin{array}{l}\text { Age } \\
\text { (years) }\end{array}$ & $\begin{array}{l}\text { Height } \\
(m)\end{array}$ & $\begin{array}{l}\text { Weight } \\
(\mathrm{kg})\end{array}$ & $F E V_{1}$ (l) & $R V($ l) & $T L C(l)$ & $R V / T L C(\%)$ & $\begin{array}{l}\text { Kco (mmol/ } \\
\mathrm{min} / \mathrm{kPa} / \mathrm{l})\end{array}$ \\
\hline $\begin{array}{l}1(\mathrm{M}) \\
2(\mathrm{M}) \\
3(\mathrm{M}) \\
4(\mathrm{M}) \\
5(\mathrm{~F}) \\
6(\mathrm{~F}) \\
7(\mathrm{M}) \\
8(\mathrm{M})\end{array}$ & $\begin{array}{l}65 \\
71 \\
50 \\
67 \\
70 \\
60 \\
72 \\
70\end{array}$ & $\begin{array}{l}1.75 \\
1.58 \\
1.64 \\
1.62 \\
1.62 \\
1.55 \\
1.65 \\
1.68\end{array}$ & $\begin{array}{l}85 \\
50 \\
78 \\
71 \\
56 \\
54 \\
58 \\
59\end{array}$ & $\begin{array}{l}0.94(-4.4) \\
0.57(-3.4) \\
0.56(-5.1) \\
0.88(-3.3) \\
0.74(-3.5) \\
0.50(-4.0) \\
0.64(-3.8) \\
0.72(-3.9)\end{array}$ & $\begin{array}{l}4 \cdot 8(5 \cdot 7) \\
3 \cdot 93(3 \cdot 8) \\
3 \cdot 19(2 \cdot 9) \\
2 \cdot 12(-0 \cdot 6) \\
4 \cdot 21(6 \cdot 2) \\
3 \cdot 41(4 \cdot 7) \\
5 \cdot 82(8 \cdot 1) \\
3 \cdot 64(2 \cdot 8)\end{array}$ & $\begin{array}{l}8 \cdot 62(2 \cdot 4) \\
5 \cdot 13(-0 \cdot 6) \\
6 \cdot 03(0) \\
5 \cdot 41(-0 \cdot 7) \\
6 \cdot 45(2 \cdot 6) \\
5 \cdot 2(1 \cdot 3) \\
8 \cdot 38(3 \cdot 2) \\
6 \cdot 2(-0 \cdot 21)\end{array}$ & $\begin{array}{l}56(3 \cdot 1) \\
77(6 \cdot 6) \\
53(3 \cdot 7) \\
39(-0 \cdot 2) \\
65(3 \cdot 9) \\
65(4 \cdot 7) \\
69(4 \cdot 9) \\
59(3 \cdot 3)\end{array}$ & $\begin{array}{l}0.73(-2.3) \\
1.09(-0.7) \\
1.28(-1.0) \\
0.99(-1.3) \\
0.82(-3.8) \\
1.07(-2.9) \\
0.83(-1.6) \\
0.68(-2.3)\end{array}$ \\
\hline
\end{tabular}

$\mathrm{FEV}_{1}=$ forced expiratory volume in one second; $\mathrm{RV}=$ residual volume; $\mathrm{TLC}=$ total lung capacity; $\mathrm{KcO}=$ carbon monoxide transfer coefficient.

\section{Methods}

Eight patients (six men) with severe COPD $\left(\mathrm{FEV}_{1}\right.$ more than three standardised residuals below predicted), aged 50-72 years and without coexistent disease, took part in the study. Signed informed consent was obtained from each patient. The study was approved by the district ethics committee. Three patients had recently undergone a course of pulmonary rehabilitation involving a programme of walking exercise. Two patients suffered deterioration of symptoms during the course of the study and testing was suspended until they returned to baseline levels.

Baseline pulmonary function tests including flow-volume curves, lung volume measurement (steady state helium dilution technique) and single breath carbon monoxide transfer factor (TLCO) were performed (PK Morgan, Kent, UK). Normal ranges were obtained using the ECSC prediction equations. ${ }^{7}$

Patients performed four incremental exercise tests over a period of four weeks (or three months in the case of the two patients with exacerbation of symptoms). The mode of exercise was randomised and all four tests were examined for between day variability. Peak values from the second test of each modality were used to compare exercise responses. In the bicycle protocol patients cycled on an electrically braked ergometer (ER 900, Mijnhardt, Holland) at a constant speed of $40 \mathrm{rpm}$ with an initial load of 10 watts. This was increased by 10 watts every minute to a symptom limited maximum. In the treadmill protocol patients performed free walking on a treadmill (Model 3060, Cambridge Instruments, UK) without using the arm supports, at a constant speed of $1.5 \mathrm{mph}$. The initial gradient was $0 \%$, increasing by $2.5 \%$ every two minutes to a symptom limited maximum. All patients were encouraged to perform to a maximum and the reason for terminating exercise was noted.

Ventilation measurements including $\mathrm{Vo}_{2}$ and $\dot{V}_{E}$ were made during rest and exercise by an integrated flow turbine system with breath by breath gas analysis averaged over eight breaths (Oxyconbeta, Mijnhardt, Holland). The FEV was measured before each test and ear lobe capillary blood samples were collected for blood gas analysis and for the determination of lactate concentration using the method described by Maughan. ${ }^{8}$ Further capillary blood samples were collected immediately after exercise for blood gas analysis and at four minutes after exercise for lactate measurement. The ECG and blood pressure were monitored throughout, as well as the arterial oxygen saturation using a pulse oximeter (Ohmeda Biox 3700 e, USA). Patients were instructed to rate breathlessness and perceived exertion during each workload by pointing to a descriptor on Borg charts. ${ }^{9}$ The Oxyconbeta system was calibrated for volume and gas analysis before each test. Measurements of oxygen consumption were expressed at STPD and ventilation at BTPS. The maximal oxygen consumption $\left(\dot{\mathrm{V}}_{2} \mathrm{max}\right)$, measured from the plateau of $\dot{\mathrm{Vo}}_{2}$ plotted against workload, is difficult to obtain in patients with COPD so the $\mathrm{VO}_{2}$ measured during the highest workload achieved was used in the analysis ( $\mathrm{Vo}_{2}$ peak).

\section{DATA ANALYSIS}

The mean and standard deviation (SD) are shown unless otherwise stated. Data from the different modes of exercise were compared using the Wilcoxon signed rank test and according to the recommendations described by Bland and Altman. ${ }^{10}$ Correlation coefficients were obtained by the least squares method. A $p$ value of $<0.05$ was considered significant.

\section{Results}

The resting pulmonary function measurements are shown in table 1 . The $\mathrm{FEV}_{1}$ was less than 1.01 in all of the patients, with the mean being $0.69(0 \cdot 16) 1$. Seven of the patients had evidence of hyperinflation, gas trapping, and a reduced TLco. The oxygen consumption, ventilation, and heart rate measurements at peak exercise for the second test of each modality are shown in table 2 , as well as the blood lactate concentrations before and after exercise. Although bicycle exercise produced a higher $\mathrm{Vo}_{2}$ peak and heart rate in four patients, and higher $\mathrm{VE}$ in six patients, there was no significant difference in $\dot{\mathrm{VO}}_{2}, \dot{\mathrm{VE}}$, and heart rate between the bicycle and treadmill at peak exercise $(p>0.05)$. However, both the lactate concentration after exercise and the increase in lactate concentration from baseline levels were significantly higher following bicycle exercise $(p<0.05)$.

The exercise duration for each type of exercise was comparable, the mean time on the bicycle being $5.02(1.8)$ minutes and on the treadmill 4.59 (3.08) minutes. However, the mean maximum workload achieved on the bicycle was 50 (19) watts, whilst the mean maximum workload on the treadmill was 33 (32) watts (calculated from body weight, velocity, sine of the gradient angle, and the acceleration due to gravity). 
Table 2 Median, mean (SD), and individual values for oxygen consumption ( $\left.\dot{V}_{2}\right)$, ventilation ( $\dot{V}_{E}$ ), and heart rate at peak workload for bicycle and treadmill (TM) exercise, lactate concentration before and four minutes after exercise and change from baseline $(\triangle)$

\begin{tabular}{|c|c|c|c|c|c|c|c|c|c|c|c|c|}
\hline \multirow[t]{3}{*}{ Subject no. } & \multicolumn{2}{|c|}{$\dot{V} \mathrm{O}_{2}$ peak $(\mathrm{ml} / \mathrm{min} / \mathrm{kg})$} & \multicolumn{2}{|c|}{$\dot{V}$ Epeak (l/min) } & \multicolumn{2}{|c|}{ Peak heart rate (beats/min) } & \multicolumn{4}{|c|}{ Lactate concentration ( $\mathrm{mmol} / \mathrm{l})$} & \multicolumn{2}{|c|}{$\triangle$ lactate concentration (mmolll) } \\
\hline & \multirow[t]{2}{*}{ Bicycle } & \multirow[t]{2}{*}{$T M$} & \multirow[t]{2}{*}{ Bicycle } & \multirow[t]{2}{*}{$T M$} & \multirow[t]{2}{*}{ Bicycle } & \multirow[t]{2}{*}{$T M$} & \multicolumn{2}{|c|}{ Bicycle } & \multicolumn{2}{|l|}{$T M$} & \multirow[t]{2}{*}{ Bicycle } & \multirow[t]{2}{*}{$T M$} \\
\hline & & & & & & & Pre & Post & Pre & Post & & \\
\hline $\begin{array}{l}1 \\
2 \\
3 \\
4 \\
5 \\
6 \\
7 \\
7 \\
8\end{array}$ & $\begin{array}{r}11 \cdot 7 \\
13.9 \\
11.7 \\
9 \cdot 3 \\
11.3 \\
15.4 \\
6.2 \\
15.0\end{array}$ & $\begin{array}{r}13 \cdot 3 \\
14 \cdot 3 \\
10 \cdot 2 \\
15 \cdot 2 \\
11 \cdot 0 \\
15 \cdot 1 \\
6 \cdot 6 \\
10 \cdot 2\end{array}$ & $\begin{array}{l}41 \cdot 1 \\
19.9 \\
23.6 \\
26.5 \\
26 \cdot 6 \\
35 \cdot 8 \\
13.5 \\
34.5\end{array}$ & $\begin{array}{l}36 \cdot 6 \\
18 \cdot 7 \\
21 \cdot 0 \\
33 \cdot 5 \\
26 \cdot 2 \\
33 \cdot 7 \\
14 \cdot 7 \\
23 \cdot 8\end{array}$ & $\begin{array}{r}115 \\
134 \\
123 \\
119 \\
118 \\
147 \\
94 \\
113\end{array}$ & $\begin{array}{r}118 \\
129 \\
109 \\
122 \\
111 \\
167 \\
98 \\
110\end{array}$ & $\begin{array}{l}0.15 \\
0.94 \\
1.43 \\
0.62 \\
0.36 \\
1.87 \\
1.2 \\
1.89\end{array}$ & $\begin{array}{l}2 \cdot 57 \\
3 \cdot 07 \\
2 \cdot 27 \\
0 \cdot 94 \\
1 \cdot 87 \\
4 \cdot 31 \\
1 \cdot 2 \\
3 \cdot 76\end{array}$ & $\begin{array}{l}0 \cdot 24 \\
0.56 \\
0 \cdot 70 \\
0.92 \\
0 \cdot 34 \\
1 \cdot 28 \\
0.80 \\
1 \cdot 67\end{array}$ & $\begin{array}{l}0.75 \\
0.93 \\
0.94 \\
1.11 \\
0.59 \\
2.93 \\
0.87 \\
1.67\end{array}$ & $\begin{array}{l}2 \cdot 42 \\
2 \cdot 13 \\
0 \cdot 84 \\
0 \cdot 32 \\
1 \cdot 51 \\
2 \cdot 44 \\
0 \cdot 00 \\
1 \cdot 87\end{array}$ & $\begin{array}{l}0.51 \\
0.37 \\
0 \cdot 24 \\
0 \cdot 19 \\
0 \cdot 25 \\
1.65 \\
0.07 \\
0.00\end{array}$ \\
\hline $\begin{array}{l}\text { Median } \\
\text { Mean } \\
\text { SD }\end{array}$ & $\begin{array}{c}11 \cdot 7 \\
11.8 \\
3.06\end{array}$ & $\begin{array}{c}12 \cdot 2 \\
12 \cdot 0 \\
3 \cdot 01\end{array}$ & $\begin{array}{r}26 \cdot 6 \\
27 \cdot 7 \\
9 \cdot 1\end{array}$ & $\begin{array}{r}25 \cdot 0 \\
26 \cdot 0 \\
7 \cdot 9\end{array}$ & $\begin{array}{l}119 \\
120 \\
15 \cdot 5\end{array}$ & $\begin{array}{l}115 \\
121 \\
21.0\end{array}$ & $\begin{array}{l}1.07 \\
1.06 \\
0.66\end{array}$ & $\begin{array}{l}2 \cdot 42 \\
2 \cdot 50 \\
1 \cdot 18\end{array}$ & $\begin{array}{l}0.75 \\
0 \cdot 81 \\
0 \cdot 48\end{array}$ & $\begin{array}{l}0.94 \\
1.22 \\
0.76\end{array}$ & $\begin{array}{l}1.69 \\
1.44 \\
0.95\end{array}$ & $\begin{array}{l}0.25 \\
0.41 \\
0.53\end{array}$ \\
\hline
\end{tabular}

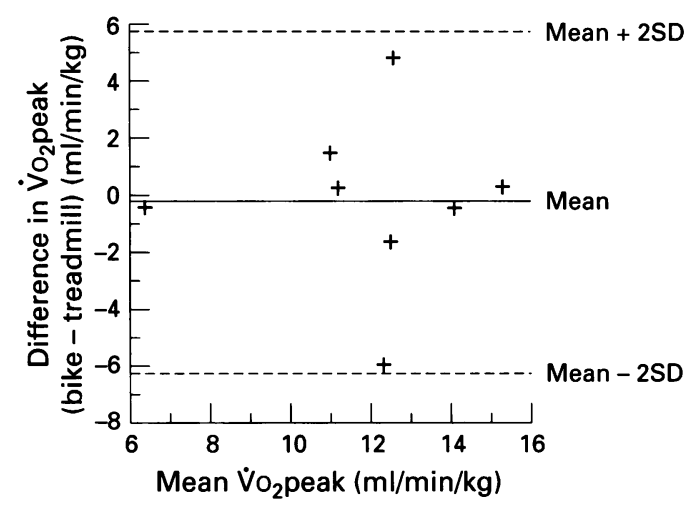

Figure 1 Differences between bicycle and treadmill oxygen consumption $\dot{V}_{2}$ peak (ml/min/kg) plotted against the mean of the two measurements.
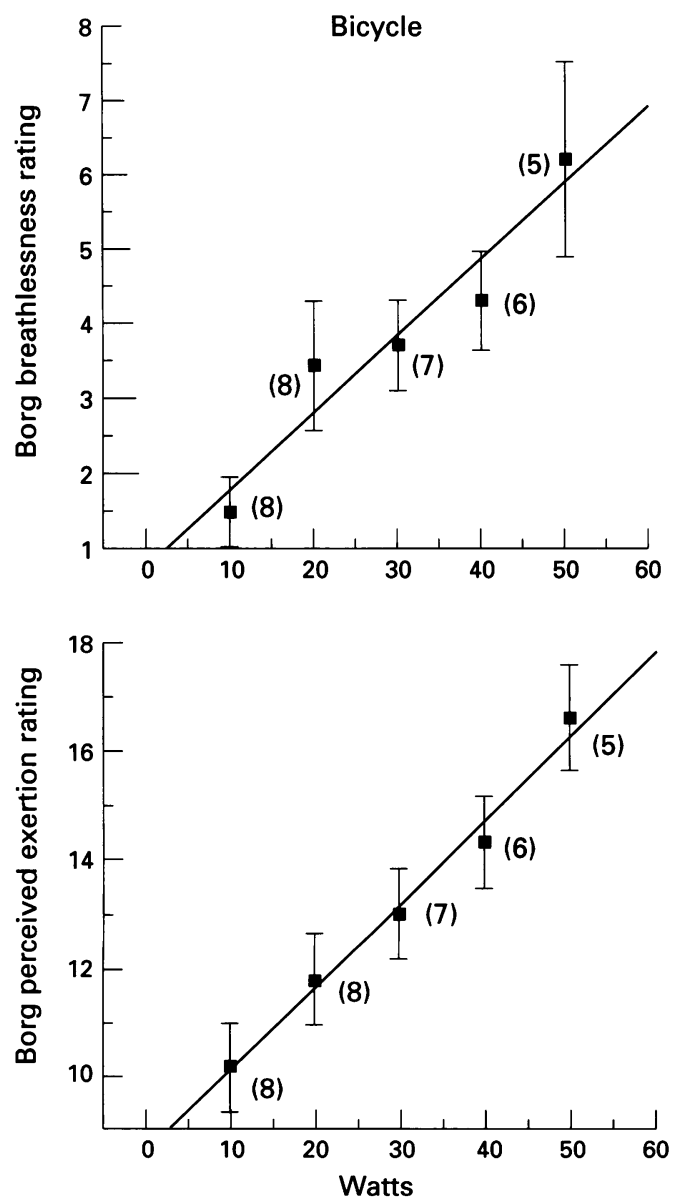

The mean between day differences for $\dot{\mathrm{Vo}}_{2}$ peak (test 2 - test 1) were 31 (155) $\mathrm{ml} /$ $\min (0.8(2.6) \mathrm{ml} / \mathrm{min} / \mathrm{kg})$ for bicycle exercise and $52(183) \mathrm{ml} / \mathrm{min}(1.0(3.3) \mathrm{ml} / \mathrm{min} / \mathrm{kg})$ for treadmill exercise. The reproducibility compares favourably with previously reported data, ${ }^{11}$ where a between day difference of 93 (81) $\mathrm{ml} / \mathrm{min}$ for bicycle exercise was measured in a group of patients with less severe COPD.

The mean of the differences between $\mathrm{Vo}_{2}$ peak for the two modes of exercise was $-0 \cdot 18(3.0) \mathrm{ml} / \mathrm{min} / \mathrm{kg}$; the range of differences is shown in fig 1 . Oxygen desaturation (fall by $>4 \%$ ) occurred in four patients during bicycle exercise and in four patients on the treadmill. There was a concurrent fall in $\mathrm{PaO}_{2}$, two patients had a slight increase in $\mathrm{PaCO}_{2}$ following
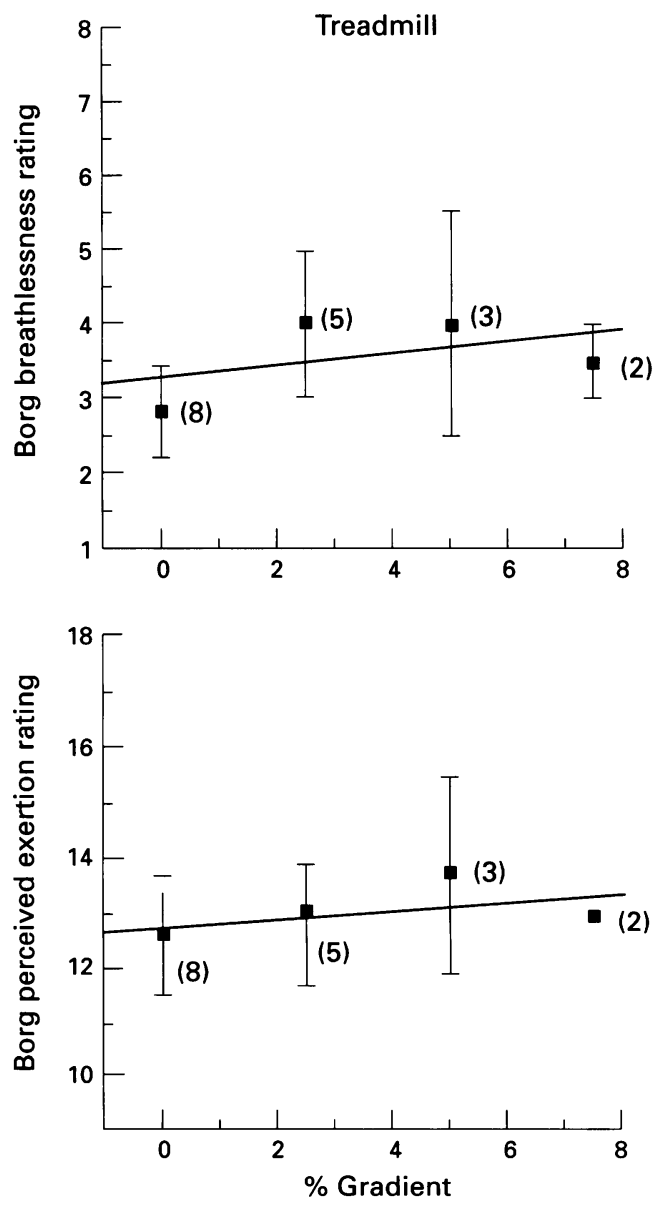

Figure 2 (Mean SE) Borg rating for breathlessness and perceived exertion for each workload increment. Numbers in parentheses are numbers of patients at each increment. 
bicycle exercise, and three patients showed similar increases following treadmill exercise.

Six patients cited dyspnoea as the reason for stopping bicycle exercise, whilst one patient cited both leg fatigue and dyspnoea, and one patient cited leg fatigue only. Five patients cited dyspnoea as the reason for stopping treadmill exercise, two patients cited leg fatigue and dyspnoea, and one patient cited leg fatigue only. Figure 2 shows the relation between the mean Borg breathlessness and exertion ratings against each workload increment for both modes of exercise. Correlation coefficients were 0.962 and 0.993 , respectively, for the bicycle and 0.477 and 0.567 , respectively, for the treadmill. Bicycle exercise produced a wider range of Borg scores and lower values of $\mathrm{Vo}_{2}$ during the initial stages of exercise.

\section{Discussion}

In normal individuals maximal oxygen consumption during treadmill exercise is generally higher than during bicycle exercise. ${ }^{412}$ In this study we found no significant difference in peak oxygen consumption, ventilation, and heart rate between the two modes of exercise. This supports work reported by Shuey et $a l^{5}$ from a study which used a steady state exercise test protocol and where the patients had less severe airways limitation (mean $\mathrm{FEV}_{1} 1 \cdot 21$ ). The mean difference between bicycle and treadmill $\mathrm{Vo}_{2}$ peak was within the $95 \%$ limits of agreement for between day reproducibility. However, individual variation was large - for example, two standard deviations for the differences between $\dot{V}_{2}$ peak measurements was $6.0 \mathrm{ml} / \mathrm{min} / \mathrm{kg}$. This suggests that the two forms of exercise cannot be used interchangeably to determine peak oxygen consumption or to follow changes in exercise capacity.

The increase in blood lactate concentration was significantly greater following the bicycle exercise. This is in contrast to the findings of Shuey et $a l^{5}$ who found no difference in the lactate concentration for the two modes of exercise. This may be accounted for by the differences in the timing of the blood collection after exercise (immediately versus four minutes after exercise), and from the smaller number of patients tested (four versus eight). In a study comparing self-paced walking on a treadmill with steady state bicycle exercise at similar levels of $\mathrm{VO}_{2}$ Cockcroft et al ${ }^{13}$ reported considerably higher levels of lactate during bicycle exercise in nine patients and higher levels of ventilation. Studies on normal subjects have shown that bicycle exercise produces a higher lactate response. Our study suggests that similar work rates were achieved as each mode of exercise produced a similar cardiorespiratory response. Whilst our calculated work rate for the treadmill was a little lower than that measured on the bicycle, this calculation is an underestimate because (1) friction is not accounted for; (2) a gradient of $0 \%$ gives a calculated work rate of 0 watts regardless of treadmill speed; (3) depending on the economy of movement the workload will be different even if the same speed and gradient is set, so workload will vary due to the different movements of running and walking; and (4) the workload depends on the material of the treadmill surface and the shoes worn. The higher lactate response for the bicycle suggests differences in the rates of formation and clearance during cycling exercise compared with walking exercise. Cycling was an unfamiliar form of exercise for these patients. The use of unfamiliar types of exercise may be the most appropriate way to elicit a lactate response in such patients where its absence precludes a patient from entering an exercise training rehabilitation programme. They might also be employed to monitor the effects of such a programme where other instruments have proved insensitive to subtle changes in overall aerobic fitness. This warrants further investigation.

Only one quarter of the patients (subjects 6 and 8) had an anaerobic threshold that could be detected by the modified V-slope method (carbon dioxide production versus $\dot{\mathrm{Vo}}_{2}$ ) described by Sue et al. ${ }^{14}$ These patients also had the highest lactate response for bicycle exercise, and this approached or exceeded the $4 \mathrm{mmol} / \mathrm{l}$ threshold level $(4.31$ and $3.76 \mathrm{mmol} / \mathrm{l}$, respectively). Alternatively, three patients had a significant increase in lactate concentration $(>2.0 \mathrm{mmol} / \mathrm{l})$ following bicycle exercise, whilst none had a significant rise following treadmill exercise. The number of patients with a detectable anaerobic threshold in this study is considerably less than the numbers reported by Sue et al who found that two thirds of the patients studied had an anaerobic threshold that could be detected by this method. This might be explained by a difference in the disease severity of the patients studied (mean $\mathrm{FEV}_{1}$ 1.231 versus 0.691 ) and the higher levels of ventilation achieved (mean $\dot{V} E$ at peak exercise, $51 \cdot 6 \mathrm{l} / \mathrm{min}$ versus $27 \cdot 7 \mathrm{l} / \mathrm{min}$ ).

The most common reason for terminating exercise was dyspnoea. This is contrary to the findings of Killian et $a l^{3}$ who found $43 \%$ of patients rated leg effort more highly than dyspnoea following bicycle exercise. The development of dyspnoea sufficient to stop exercising before the onset of leg fatigue reflects the markedly diminished ventilatory reserves found in this group. The wider range of Borg scores and lower $\mathrm{VO}_{2}$ measurements during the initial stages of bicycle exercise suggest that a more gentle introduction is achieved by supporting the body weight. The stronger association between these variables is reflected in the higher correlation coefficients for the bicycle compared with the treadmill.

The small number of patients studied reflects the difficulty in carrying out a study of this type, where sufficient numbers still able to perform some exercise and remain stable throughout the study period are difficult to recruit. Although the number of patients studied was small, there were no clear differences between the ventilation and heart rate responses to the two modes of exercise. Individual variability was large, however, therefore the best exercise performance in this type of patient 
cannot be predicted and the two forms of exercise are not interchangeable. The highest $\dot{\mathrm{Vo}}_{2}$ might only be determined if both types of test were carried out. These results do not support the hypothesis that patients with severe COPD will achieve a better performance during exercise which provides support for the upper limb muscles than at unsupported exercise. Nevertheless, bicycle exercise produced similar $\dot{V}{ }_{2}$ peak measurements, a greater lactate response, and a more gentle introduction compared with treadmill exercise.

RSM was sponsored by the Raj Nanda Pulmonary Disease Research Trust, Delhi and the British Thoracic Society.

1 Dillard TA. Prediction of ventilation at maximal exercise in patients with chronic airflow limitation. Chest 1987;92: 195-6.

2 Dillard TA, Piantadosi S, Rajagopal KR. Prediction of ventilation at maximal exercise in chronic airflow obventilation at maximal exercise in chronic

3 Killian KJ, Le Blanc P, Martin DH, Summers E, Jones NL, Campbell EJM. Exercise capacity and ventilatory, circulatory and symptom limitation in patients with chronic airflow limitation. Am Rev Respir Dis 1992;146: 935-40.

4 Shepherd RJ. Standard tests of aerobic power. In: Shepherd
RJ, ed. Frontiers in fitness. Springfield IL: Charles C Thomas, 1971 .

5 Shuey CB Jnr, Pierce AK, Johnson RL Jnr. An evaluation of exercise tests in chronic obstructive lung disease. $\mathcal{F} A p p l$ Physiol 1969;27:256-61.

6 Criner GJ, Celli BR. Effect of unsupported arm exercise on ventilatory muscle recruitment in patients with severe chronic airflow obstruction. Am Rev Respir Dis 1988;138: 856-86.

7 Quanjer $\mathrm{PhH}$, ed. Standandization of lung function tests 1993 update. Report of Working Party for the European Community for Steel and Coal. Eur Respir f 1993;6(suppl): 16.

8 Maughan R. A simple rapid method for the determination of glucose, pyruvate, alanine, 3-hydroxybutyrate and acetoacetate on a single $20 \mu \mathrm{l}$ blood sample. Clin Chim Acta 1982;122:231-40.

9 Borg GAV. Psychophysical basis of perceived exertion. Med Sci Sports Exerc 1982;14:377-81.

10 Bland MJ, Altman DG. Statistical methods for assessing agreement between two methods of clinical measurement. Lancet 1986; i:307-10.

11 Brown SE, Fishcer CE, Stansbury DW, Light RW. Reproducibility of $\mathrm{Vo}_{2} \mathrm{max}$ in patients with chronic airflow obstruction. Am Rev Respir Dis 1985;131:435-8.

12 Withers RT, Sherman WM, Miller JM, Costello DL. Specificity of anaerobic threshold in endurance trained cyclists ficity of anaerobic threshold in endurance trained

13 Cockcroft A, Beaumont A, Adams L, Guz A. Arterial oxygen desaturation during treadmill and bicycle exercise in patients with chronic obstructive airways disease. Clin $S c i$ 1985;68:327-32.

14 Sue DY, Wasserman K, Bruce Moricca R, Casaburi R. Metabolic acidosis during exercise in patients with chronic obstructive pulmonary disease. Chest 1988;94:931-8. 